the same in both cases. As a consequence of this, the difference of the numerators will now be the same as the difference of the denominators, viz., 1 in this case. We wish it to be 15 , and therefore multiply by 15 all through to obtain the original and final ages.

Similarly with the general case, "two numbers are in the ratio of $a$ to $b$. If $n$ be added to each, the ratio becomes that of $c$ to $d$. Find the original and final numbers."

$$
\begin{aligned}
\frac{a}{b} & , \frac{c}{d} \\
\frac{a(c-d)}{b(c-d)} & , \quad \frac{c(a-b)}{d(a-b)} \\
\frac{a(c-d) \frac{n}{a d-b c}}{b(c-d) \frac{n}{a d-b c}} & , \frac{c(a-b) \frac{n}{a d-b c}}{d(a-b) \frac{n}{a d-b c}} .
\end{aligned}
$$

The four numbers in the last line sre the numbers required.

\title{
John Dougall.
}

The Equivalence of certain Simultaneous Equations. -The object of this note will, perhaps, be best explained by first repeating the usual steps in the solution of two simultaneous equations in $x, y$, where one is linear and the other is of the second degree. Let the equations be

$$
\begin{array}{r}
x-y+1=0, \ldots \ldots . \\
2 x^{2}-y^{2}-7 x+5 y-1=0 .
\end{array}
$$

From (1) we obtain $y=x+1$. Substituting $y=x+1$ in (2) we next obtain

$$
x^{2}-4 x+3=0,
$$

whence

$$
x=1 \text { or } 3 \text {. }
$$

Going back to (1), we find that

$$
\begin{aligned}
& \text { if } x=1, y=2 ; \\
& \text { if } x=3, y=4 .
\end{aligned}
$$

The solutions are therefore $(1,2),(3,4)$. 
THE gQUIVALENCE OF CERTAIN SIMULTANEOUS EQUATION8.

Referring to the step printed in italics, we wish in this note to call attention to why we go back to (1) and not to (2).

According to the Remainder Theorem, the characteristic of equation (3) is the Remainder when

$$
2 x^{2}-y^{2}-7 x+5 y-1
$$

regarded as a trinomial in $y$, is divided by

$$
x-y+1
$$

regarded as a binomial in $y$, so that (2) may, by division, be put in the form

$$
(x-y+1)(x+y-4)+\left(x^{2}-4 x+3\right)=0 .
$$

Hence we see that the system

$$
\left\{\begin{array}{r}
x-y+1=0 \ldots \ldots \\
2 x^{2}-y^{2}-7 x+5 y-1=0
\end{array}\right.
$$

is equivalent to the system

$$
\left\{\begin{array}{r}
x-y+1=0 \ldots \ldots \ldots \ldots \ldots \ldots \\
(x-y+1)(x+y-4)+\left(x^{2}-4 x+3\right)=0
\end{array}\right.
$$

and therefore to the system

$$
\left\{\begin{array}{c}
x-y+1=0 \\
x^{2}-4 x+3=0
\end{array}\right.
$$

and therefore to the systems

$$
\left\{\begin{array}{r}
x-y+1=0 \\
x-1=0
\end{array} ; \quad\left\{\begin{array}{r}
x-y+1=0 \\
x-3=0
\end{array}\right.\right.
$$

and finally to

$$
\left\{\begin{array}{l}
x=1 \\
y=2
\end{array} ; \quad\left\{\begin{array}{l}
x=3 \\
y=4
\end{array}\right.\right.
$$

If we go back to (2) instead of to (1), we get the system

$$
\left\{\begin{array}{r}
2 x^{2}-y^{2}-7 x+5 y-1=0 \\
x^{2}-4 x+3=0
\end{array}\right.
$$

which is equivalent to

$$
\left\{\begin{array}{c}
(x-y+1)(x+y-4)+\left(x^{2}-4 x+3\right)=0 \\
x^{2}-4 x+3=0
\end{array}\right.
$$


MATHEMATICAL NOTES.

and therefore to

$$
\left\{\begin{array}{c}
(x-y+1)(x+y-4)+\left(x^{2}-4 x+3\right)=0 \\
(x-y+1)(x+y-4)=0 ;
\end{array}\right.
$$

and hence to the systems

$$
\left\{\begin{array}{rl}
2 x^{2}-y^{2}-7 x+5 y-1 & =0 \\
x-y+1 & =0
\end{array} ; \quad\left\{\begin{aligned}
2 x^{2}-y^{2}-7 x+5 y-1 & =0 \\
x+y-4 & =0
\end{aligned}\right.\right.
$$

so that an extraneous system is introduced.

John E. RidLey. 